An increasing number of patients with motor neurone disease (MND) in the UK and Ireland are using assisted ventilation to control symptoms and prolong life. A small proportion of these are initiated on long term tracheostomy ventilation (TV). NICE guidelines dictate that MND patients should routinely receive specialist palliative care input, yet research into the management of TV MND patients in the hospice setting is limited. Objective

The aim of this study was to explore the extent to which hospices and specialist palliative care units (SPCUs) in the UK and Ireland currently manage MND patients using assisted ventilation, especially TV.

Methods A literature review was used to guide the development of an electronic questionnaire which was sent to medical directors or lead physicians in all hospices/specialist palliative care units in the United Kingdom and Republic of Ireland. the questionnaire was sent to 184 units.

Results The response rate was 41.3\% $(n=76)$. 97\% units admit MND patients on non-invasive ventilation (NIV) but only 33\% admit TV MND patients $(n=22)$. However $40 \%$ of respondents indicated that community palliative care support was provided to TV MND patients. 61 respondents indicated adequate expertise in the management of NIV, while only 6 indicated adequate expertise in managing TV. While the majority of respondents (96\%) indicated that Advance care planning was routinely offered to the MND patients, less than half routinely included conversations regarding ITU admission (40\%) or invasive ventilation (39\%). Only $11(14 \%)$ of respondents indicated having personal experience of withdrawal of TV in MND patients.

Conclusion This study has highlighted a perceived lack of expertise in the management TV MND patients within hospices/SPCUs with less than half of units allowing admission for TV MND patients. There is a need for further research in this area.

\section{EVALUATION OF CAN-GUIDE (CANCER-GOAL USE IN DECISIONS): A DECISION MAKING SUPPORT PACKAGE TO ENHANCE SHARED DECISION MAKING IN PROGRESSING CANCER}

Grant Punnett, Leanne Howieson, Charlotte J Heaven, Sally Taylor, Carole Mula, Wendy Makin, Janelle Yorke. The Christie NHS Foundation Trust, University of Manchester

\subsection{6/bmjspcare-2018-ASPabstracts. 190}

Introduction National guidelines recommend that shared decision making be employed where several treatment or supportive care options are available to the patients with cancer. Shared decision making is associated with positive outcomes for patients including reduced decision regret surrounding their treatment and reduction of unrealistic expectations. Optimising shared decision making in situations where cancer is no longer curable may facilitate the discussion about not only the aims of treatment but also the patient's life goals and how their treatment options may affect these.

Aim to evaluate the Cancer-Goal Use In DEcisions (CanGUIDE) web-based communication/decision aid as a resource for facilitating goals of care conversations and shared decision making in the context of incurable cancer.

Method A pre-test post-test pilot study to assess the benefit of Can-GUIDE on patient experience and shared decision making. The quality of the decision making process is currently being assessed by determining the effect on patient decision self-efficacy, decision conflict, preparation for decision making and perceived involvement in shared decision making with their clinician. Oncology clinician opinion will be sought to determine whether patient responses to the package helped guide GOC conversations within their consultations. Furthermore, GPs will be surveyed to determine the usefulness of receiving a tool documenting GOC conversations within oncology consultations. The views of all participant groups surrounding the usability and acceptability of Can-GUIDE are also being investigated.

Results Currently, 14 patients have been recruited for the study with 4 withdrawn due to progressing disease or noncompliance with protocol. Data collection is due to complete March 2018 with a total of 40 patients recruited.

Conclusions If our pilot results demonstrate the potential value of Can-GUIDE a fully powered randomised controlled trial will be conducted to tests its effectiveness on improving patient-centred outcomes.

\section{BEYOND EARLY INTERVENTION - INTEGRATED WORKING IN A BRAIN TUMOUR CLINIC} ${ }^{1,2}$ Carolyn Campbell, ${ }^{3}$ Josephine Peck, ${ }^{3}$ Victoria Teague, ${ }^{2}$ Fiona Minear, ${ }^{2}$ Glad Baldry,
1,2 Jane Gibbins, ${ }^{1}$ Deborah Stevens. ${ }^{1}$ Cornwall Hospice Care; ${ }^{2}$ Royal Cornwall Hospitals
Trust; ${ }^{3}$ University of Exeter Medical School

10.1136/bmjspcare-2018-ASPabstracts. 191

Background An increasing body of evidence supports early palliative care involvement in oncology as improving quality of life, advance care planning and decision making. Patients with brain tumours experience high symptom, social and psychological burden and those with lower grade tumours may struggle to access palliative care services earlier in their disease trajectory. This tumour site has not been studied in any previous work on early intervention or joint working. This service review looks at a joint oncology/palliative care, consultant delivered, brain tumour clinic.

Methods Records of 133 consecutive consultations (82 patients) were analysed for demographics, diagnosis, clinician seen, primary purpose of appointment and additional topics addressed. For a cohort of patients from each diagnostic group, all consultations since diagnosis were reviewed to establish any patterns of engagement over time and disease progression.

Results $43.6 \%$ of consultations were with the palliative care consultant (PCC). Longitudinally, $81.8 \%$ of patients saw the PCC at least once. Whilst PCC (60.3\%) and oncologist (62.6\%) had a similar proportion of complex consultations (those dealing with at least one additional topic) for the oncologist these were concentrated in new patient consultations and treatment decisions (63\%) and for patients with glioblastomas (60.1\%); whereas for the PCC, complex consultations were distributed across all tumour types except pituitary and predominantly occurred in routine reviews (76.5\%). Levels of complexity were comparable in patients with lower grade gliomas or meningioma to those with glioblastomas.

Conclusions This analysis demonstrates a high level of complex need in patients with brain tumours and particularly for those with lower grade tumours over the very prolonged course of their disease. Integrated working appears to facilitate early involvement of palliative care and collaborative care, 
with patients accessing the most appropriate clinician for their current needs.

\section{Symptom management | Posters $165-177$}

\section{IMPROVING CARE OF PATIENTS WITH TERMINAL AGITATION (TA) AT END OF LIFE AT LEEDS TEACHING HOSPITALS NHS TRUST}

Claire Iwaniszak, Elizabeth Rees, Rachael Meal, Anna Winfield, Craig Pattison, Katie Hodge, Suzanne Kite. Leeds Teaching Hospitals

\subsection{6/bmjspcare-2018-ASPabstracts. 192}

Aim Our multidisciplinary team quality improvement project aimed to ensure that all dying patients who experience TA have an effective individualised plan of care on an acute stroke ward (L21) within a tertiary centre.

Background Leeds Teaching Hospitals was selected to be one of 10 hospitals to be part of the national 'Building on the Best Programme' in conjunction with Hospice UK, Macmillan, NHSE and NHSI. This was to build on what was already being achieved by hospitals to improve the quality of end of life care (EOLC). Often improvement work focuses on the management of pain and we wanted to focus on improving the care of patients with TA, a symptom found in up to $90 \%$ of dying patients, but not always focused on.

Methods This work was led by the Palliative Care team in conjunction with the frontline team on L21. A driver diagram was produced to identify key areas of focus (FIG 1). Interventions included ward-based role modelling of expert care of the dying; (this included the recognition, assessment, care planning and evaluation of interventions for patients experiencing TA), ward-based teaching, display and presentation of improvement data and discussion of dying patients in safety huddles. Run charts were created for these initiatives, with baseline data pre-intervention and on-going data collection during the testing, implementation and sustainability phases. All interventions were developed following identification of gaps in care delivery/evaluation.

Results Run charts (FIG 2-4) demonstrated statistically significant improvements in the rate of assessment, reassessment and evaluation of terminal agitation $(p<0.05)$. Routine review and dissemination of data with the frontline teams in these initiatives enhanced collaborative engagement, motivation and success.

Conclusion Through collaborative working and ward-based role modelling we have demonstrated it is possible to improve the overall management of this challenging symptom in terminal care.

\section{PUT UP WITH DYSGEUSIA... I DON'T 'ZINC' SO!}

Juliet McCormack, Matthew Curtis. Southern Health NHS Foundation Trust

\subsection{6/bmjspcare-2018-ASPabstracts. 193}

Background Taste or gustatory dysfunctions are implicated in loss of appetite, unintended weight loss, malnutrition and reduced quality of life. The benefits of Zinc supplementation for dysgeusia have been documented for the past 20 years with moderate quality supportive evidence available.
Improvements in taste have been demonstrated in patients receiving chemoradiotherapy and in those with idiopathic taste loss. However, this approach does not appear to be part of routine practice.

Aims We present a case study of a patient experiencing dysgeusia who was successfully treated with zinc supplementation and lifestyle changes.

Case study description 60 year old patient with a uterine carcinoma receiving Sirolimus treatment. She described extreme taste sensation changes within 1 month of commencing the Sirolimus; foods tasted too salty with an accompanying overwhelming metallic taste. This severely impacted upon her enjoyment of food with reduced appetite, associated weight loss, fatigue and social withdrawal.

Physical examination of the oral cavity showed no evidence of infection, ulcers or candidiasis. Zinc supplementation was commenced alongside routine advice including regular oral hygiene, dietary changes and cooking techniques.

Results Specific taste change improvements occurred within 3 weeks with reduction in the salty and metallic tastes. This resulted in improved appetite and tolerance of a wider variety of foods. During subsequent weeks, alongside immunotherapy, there was continued improvement in appetite, enjoyment of eating and quality of life.

Conclusion Although not routine clinical practice, this case demonstrates how inexpensive mineral supplementation can improve dysgeusia and supported tolerance of immunotherapy.

Based on this case, we plan to review our palliative care caseload to identify prevalence of this symptom. Our intention would be to then develop an approach to further research this intervention. The research hypothesis is that zinc supplementation plus lifestyle changes is superior to lifestyle changes alone for dysgeusia in palliative care.

\section{METHYLPHENIDATE FOR DEPRESSION IN PALLIATIVE CARE - WHAT'S NEW?}

Angela Star, Bridget Candy, Louise Jones, Adrian Tookman, Michael King, Paddy Stone. Marie Curie Palliative Care Research Unit, Department of Mental Health Sciences, Royal Free \& University College Medical School

\subsection{6/bmjspcare-2018-ASPabstracts. 194}

Background Symptoms of depression (SDEP) are common; antidepressants are not always effective or appropriate, especially in palliative care (PC) cohorts (where the priority is timely effect). Methylphenidate, a psychostimulant, may offer an alternative. The related 2008 Cochrane review ${ }^{1}$ contained a heterogeneous group of trials and was inconclusive. New trial data has become available.

Aim To evaluate via systematic review the effectiveness and safety of methylphenidate (single-agent or adjunct) in treating depression and SDEP in adults who have advanced medical conditions or are receiving PC.

Methods We searched published papers of randomised controlled trials (RCTs) in any language from 2006-present on key online databases, manufacturer's trial listings, reference searching, and personal communication. Citations were screened in duplicate. Trials meeting criteria were collated with relevant RCTs from the previous review. ${ }^{1}$ Cohorts with traumatic brain injury were excluded.

The primary outcome measures were: effect of MPD on overall SDEP (at days 7 and 28), and adverse events (AEs). Where homogeneity allowed, meta-analysis was planned. 